\title{
Colectomia Direita Associada à Gastroduodenopancreatectomia em Bloco por Tumor Infiltrativo de Colon - Relato de Caso
}

\author{
Right Colectomy Associated to Gastroduodenopancreatectomy in Bloc for \\ Infiltrative Colon Tumor - Case Report
}

\author{
MARIA AUXILIADORA PROLUNGATTI CESAR ${ }^{1}$; DEOMIR GERMANO BASSI ${ }^{2}$; PEDRO ROBERTO DE PAULA ${ }^{3}$; \\ PRISCILATEIXEIRAALVES ${ }^{4}$;EDUARDOFORTES DEALBUQUERQUE ${ }^{5}$
}

\begin{abstract}
${ }^{1}$ Professor Assistente Doutor do Departamento de Medicina da UNITAU, Mestre e Doutora em Medicina pela Faculdade de Ciências Médicas da Santa Casa de São Paulo; ${ }^{2}$ Professor Titular do Departamento de Medicina da UNITAU, Mestre

e Doutor pela Universidade Federal de São Paulo; ${ }^{3}$ Professor Assistente Doutor do Departamento de Medicina da UNITAU, Mestre e Doutor pela Universidade Federal de São Paulo; ${ }^{4}$ Residente de Segundo Ano do Serviço de Clínica Cirúrgica do Hospital Universitário de Taubaté; ${ }^{5}$ Residente de Segundo Ano do Serviço de Clínica Cirúrgica do Hospital Universitário de Taubaté.
\end{abstract}

\begin{abstract}
CESAR MAP; BASSI DG; PAULA PR; ALVES PT; ALBUQUERQUE EF. Colectomia Direita Associada à Gastroduodenopancreatectomia em Bloco por Tumor Infiltrativo de Colon - Relato de Caso. Rev bras Coloproct, 2009;29(3): 386-388.

RESUMO: INTRODUÇÃO: A eficácia da ressecção em bloco duodenopancreático para tumores infiltrativos não periampulares ainda não está bem definida ${ }^{3}$, mas sabe-se que em tumores colorretais localmente avançados, a ressecção multivisceral é, na maioria das vezes, a chance de melhor qualidade de vida ${ }^{4}$. OBJETIVO: apresentar um caso de neoplasia de cólon direito com invasão duodenopancreática tratado com gastroduodenopancreatectomia. RELATO DE CASO: Paciente de 73 anos, branco, que há oito meses apresentava astenia, adinamia e emagrecimento de aproximadamente $20 \mathrm{~kg}$. Ao exame físico: IMC: 20,3, descorado $++/ 4$. Abdome com massa palpável em flanco direito de aproximadamente $15 \mathrm{~cm}$. A colonoscopia demonstrou tumor de cólon direito e o anátomo-patológico evidenciou adenocarcinoma moderadamente diferenciado. A Tomografia computadorizada demonstrou os vasos mesentéricos livres de invasão. No intra-operatório se observou massa tumoral envolvendo cólon ascendente até ângulo hepático invadindo segunda para terceira porção duodenal. Foi realizada colectomia direita associada à gastroduodenopancreatectomia em bloco. $O$ anátomo-patológico da peça revelou adenocarcinoma moderadamente diferenciado de intestino grosso com metástases em tecido duodenal e pancreático com infiltração angio-linfática. $O$ paciente sobreviveu por 8 meses. Conclusão: A gastroduodenopancreatectomia deve ser considerada para tumores não periampulares na ausência de metástases à distância e quando a condição clinica do paciente permitir e foi a conduta adequada para este paciente.
\end{abstract}

Descritores: Cólon Ascendente, Cirurgia Colorretal, Pancreatectomia.

\section{INTRODUÇÃO}

A gastroduodenopancratectomia (GDP) é freqüentemente realizada para tumores malignos periampulares, tendo o seu papel reconhecido na melhora da sobrevida em comparação com os procedimentos paliativos ${ }^{1}$. A ressecção de alguns tumores metastáticos colorretais já está bem estabelecida, como é o caso das ressecções hepáticas e pulmonares ${ }^{2}$. Entretanto a eficácia da ressecção em bloco duodenopancreático para tumores infiltrativos não periampulares ainda não está bem definida ${ }^{3}$, mas sabe-se que em tumores colorretais localmente avançados, a ressecção multivisceral é, na maioria das vezes, a chance de melhor qualidade de vida ${ }^{4}$.

Trabalho realizado no Hospital Universitário de Taubaté Serviço de Clínica Cirúrgica.

$\overline{\text { Recebido } 21 / 11 / 2008}$

Aceito para publicação em 29/12/2008 
Colectomia Direita Associada à Gastroduodenopancreatectomia em Bloco por Tumor Infiltrativo de Cólon - Relato de Caso Maria Auxiliadora Prolungatti Cesar e Cols.

\section{OBJETIVO}

Apresentar um caso de tumor de cólon direito com invasão duodenopancreatica por contiguidade em que foi realizada a gastroduodenopancreatectomia.

\section{RELATO DE CASO}

Paciente de 73 anos, que há oito meses apresentava astenia, adinamia, dor epigástrica acompanhada de vômitos pós-prandiais e emagrecimento de 20 kg. Negava alteração do ritmo intestinal. Tabagista e etilista.

Ao exame físico: IMC: 20,3, descorado $++/ 4$. Ao exame abdominal presença de massa palpável em flanco direito com $15 \mathrm{~cm}$. A colonoscopia demonstrou tumor de cólon direito e o anatomo-patológico evidenciou adenocarcinoma moderadamente diferenciado. A tomografia computadorizada de abdome e pelve evidenciou tumoração em cólon ascendente provocando estreitamento da luz e infiltrando a gordura pericólica (Figura 1). Ausência de linfonodos acometidos. Acometimento próximo a veia cava inferior, porém com vasos mesentéricos aparentemente livres. Ausência de líquido livre ou imagens sugestivas de metástases hepáticas

No intra-operatório houve o diagnóstico de envolvimento duodenopancreático com massa tumoral envolvendo cólon ascendente até ângulo hepático invadindo segunda e terceira porção duodenal e a endoscopia intra-operatória demonstrou lesão infiltrativa acometendo segunda porção duodenal ocupando $30 \%$ da sua luz, em face posterior, $\operatorname{com} 3,0 \mathrm{~cm}$.

A duração do ato cirúrgico foi de $350 \mathrm{~min}$. Realizou-se colectomia direita associada à gastroduodenopancreatectomia em bloco (Figura 2). A anastomose com o pâncreas foi por telescopagem com alça jejunal em dois planos, a do colédoco com o jejuno término-lateral em plano único total, a anastomose do estômago término-lateral na alça jejunal em dois planos e a anastomose ileotransverso em dois planos.

Evoluiu com coleção abdominal em flanco D, que foi drenada por punção guiada por TC no $11^{\circ} \mathrm{e}$ repetida nos $20^{\circ}$ e $28^{\circ}$ dias de pós-operatório (PO). Teve alta no $40^{\circ} \mathrm{PO}$, sendo encaminhado para oncologia clínica com uma sobrevida pós-operatória de oito meses. O anatomopatológico da peça revelou adenocarcinoma moderadamente diferenciado de in- testino grosso com invasão tumoral em tecido duodenal e pancreático. Presença de infiltração angio-linfática.

\section{DISCUSSÃO}

A GDP é um procedimento realizado frequentemente para tumores malignos periampulares, tendo seu benefício na sobrevida e uma menor morbi-mortalidade operatória em centros com experiência nesse tipo de procedimento ${ }^{1}$. Sendo muitas vezes a ressecção multivisceral de tumores colorretais localmente avançados a chance de melhora da sobrevida do paciente a gastroduodenopancreatectomia vem contribuir para o tratamento, mesmo que paliativo, da doença ${ }^{3,4}$.

Nosso paciente apresentava um quadro clínico insidioso, como é característico de tumor de cólon direito e ceco, com exames de estadiamento não evidenciando acometimento metastático, mas com evidência de grande massa abdominal e diagnóstico pela colonoscopia de tumoração estenosante em transverso. O achado intra-operatório de invasão duodenal e

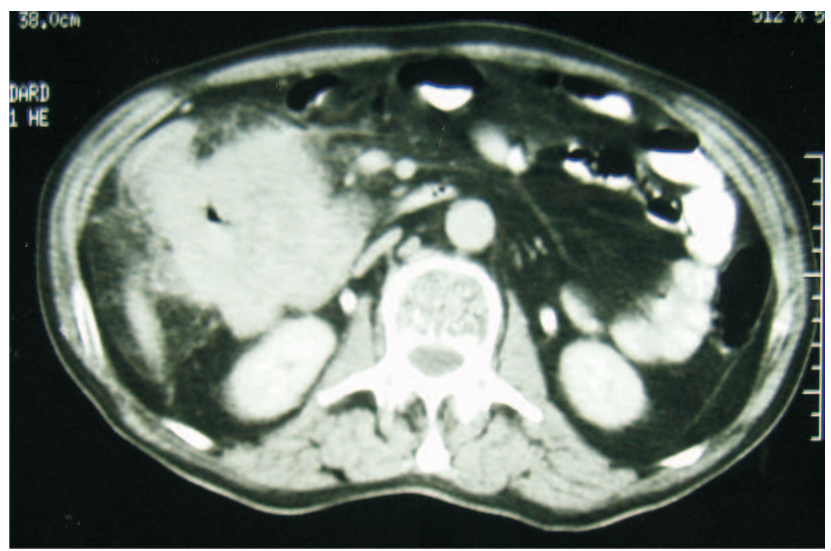

Figura 1 - Neoplasia em fossa ilíaca direita.
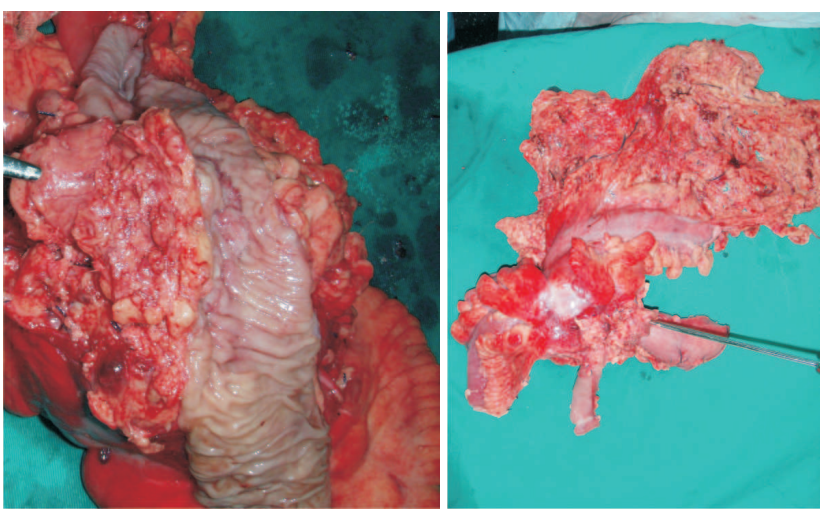

Figura 2 - Produto de gastroduodenopancreatectomia. 
Rev bras Coloproct Julho/Setembro, 2009
Colectomia Direita Associada à Gastroduodenopancreatectomia em Bloco por Tumor Infiltrativo de Cólon - Relato de Caso Maria Auxiliadora Prolungatti Cesar e Cols.
Vol. 29 pancreática nos conduziu a ressecção dessas estruturas juntamente com a tumoração, permitindo assim a retirada da lesão com margens não comprometidas como evidenciou o resultado do estudo anatomo-patológico da peça. Tal procedimento permitiu uma melhora da sobrevida de nosso paciente que foi encaminhado para quimioterapia e teve uma sobrevida pós-operatória de 8 meses.

Em casuística com 11 pacientes portadores de tumores não periampulares submetidos a GDP foi encontrada média de sobrevida de 38 meses $^{3}$. É descrito também uma média de sobrevida de 24 meses após ressecção pancreática, devido a metástases isolada em pâncreas de melanomas ${ }^{5}$. Uma revisão sobre carcinoma de células renais metastático isoladamente para pâncreas, submetidos à GDP, descreve uma média de sobrevida de 14 meses $^{3}$.
Dessa maneira, achamos que a gastroduodenopancreatectomia deve ser considerada para tumores não periampulares, principalmente em pacientes com ausência de metástases à distância e quando sua condição clinica permitir. A GDP para tumores não periampulares é factível e a ressecção em bloco, sobretudo para os tumores colorretais, é chance de controle da doença neoplásica e aumento da sobrevida.

\section{CONCLUSÃO}

A gastroduodenopancreatectomia foi a conduta adequada para esse paciente e pode ser considerada opção para pacientes com neoplasia avançada de cólon com invasão de estruturas adjacentes.

\begin{abstract}
INTRODUCTION: The effectiveness of duodenopancreatic ressection in block for infiltrative periampolar tumors are not yet know, but it is known that in local advanced colorretais tumors, the multivisceral ressection is, most of the time, the possibility of better quality of life. OBJECTIVE: To present a case of right colon neoplasm with duodenopancreatic invasion treated with gastroduodenopancreatectomy. CASE REPORT: Man, 73 years old, white, that for eight months presented asthenia,and lost approximately $20 \mathrm{~kg}$. Physical examination: mass in right flank of approximately $15 \mathrm{~cm}$. The colonoscopy demonstrated right tumor of colon, adenocarcinoma. Durin a surgery it was observed ascending tumoral mass involving colon until hepatic angle invading the second to the third duodenal portion. Right colectomy associated with gastroduodenopancreatectomy was performed. The pathological exam differentiated adenocarcinoma of duodenal and pancreatic thick intestine with metastasis and angiolymphatic infiltration. The patient survived for 8 months. CONCLUSION: The gastroduodenopancreatectomy must be considered the absence of distant metastasis and when the clinical condition of the patient not to allow and was the behavior adjusted for this patient.
\end{abstract}

Key words: Ascending Colon, Colorretal Surgery, Pancreatectomy.

\section{REFERÊNCIAS}

1. Lieberman MD; KIlburn H;Lindsey M; Brennan MF. Relation of perioperative deaths to hospital volume among patients undergoing pancreatic resection for malignancy. Ann Surg. 1995; 222(5): p638-45.

1. Harrison LE; Merchant N; Cohen AM; Brennan MF. Pancreaticoduodenectomy for nonperiampullary primary tumors. Am J Surg. 1997; 174(4): p393-5.

2. Carvalho GSS; Mali Jr J; Dias JÁ; Albagli R. Gastroduodenopancreatectomia ampliada para tumores não periampulares. Rev. Col. Bras. Cir. [periódico na Internet] 2007; 34(2). Disponível em URL: http:// www.scielo.br/rcbc.
3. Vieira RA; Lopes A; Almeida PA; Rossi BM; Nakagawa WT; Ferreira FO; Melo CA. Prognostic factors in locally advanced colon câncer treated by extended resection. Rev. Hosp. Clin. Fac. Med. São Paulo. 2004; 59(6): p361-368.

4. Wood TF; DiFronzo LA; Rose DM ; Haigh P I ; Stern S 1 ; Wanek 1; Essner R ; Morton D L Does complete resection of melanoma metastatic to solid intra-abdominal organs improve survival? Ann Surg Oncol. 2001; 8: p658-62.

Endereço para correspondencia: MARIA AUXILIADORA PROLUNGATTI CESAR

Serviço de Clínica Cirúrgica - Hospital Universitário de Taubaté Avenida granadeiro Guimarães, 270

Taubaté - SP 